\title{
Carrageenan-Based Hydrogels for the Controlled Delivery of PDGF-BB in Bone Tissue Engineering Applications
}

\author{
Vítor Espírito Santo, ${ }^{*,+\neq}$ Ana M. Frias, ${ }^{\dagger, \ddagger}$ Michela Carida, ${ }^{\S}$ Ranieri Cancedda, ${ }^{\S, \perp}$ \\ Manuela E. Gomes, ${ }^{\mathrm{t}, \neq}$ João F. Mano, ${ }^{\dagger, \ddagger}$ and Rui L. Reis ${ }^{\dagger, \neq}$

\begin{abstract}
3B's Research Group - Biomaterials, Biodegradables and Biomimetics, University of Minho, Headquarters of the European Institute of Excellence on Tissue Engineering and Regenerative Medicine, Ave Park, S. Cláudio do Barco, 4806-909 Taipas, Guimarães, Portugal, Institute for Biotechnology and Bioengineering, PT Government Associated Laboratory, Braga, Portugal, Dipartimento di Oncologia, Biologia e Genetica, Università di Genova, Genova, Italy, and Istituto Nazionale per la Ricerca sul Cancro, Genova, Italy
\end{abstract}

Received December 24, 2008; Revised Manuscript Received April 2, 2009

\begin{abstract}
One of the major drawbacks found in most bone tissue engineering approaches developed so far consists in the lack of strategies to promote vascularisation. Some studies have addressed different issues that may enhance vascularisation in tissue engineered constructs, most of them involving the use of growth factors (GFs) that are involved in the restitution of the vascularity in a damaged zone. The use of sustained delivery systems might also play an important role in the re-establishment of angiogenesis. In this study, $\kappa$-carrageenan, a naturally occurring polymer, was used to develop hydrogel beads with the ability to incorporate GFs with the purpose of establishing an effective angiogenesis mechanism. Some processing parameters were studied and their influence on the final bead properties was evaluated. Platelet derived growth factor (PDGF-BB) was selected as the angiogenic factor to incorporate in the developed beads, and the results demonstrate the achievement of an efficient encapsulation and controlled release profile matching those usually required for the development of a fully functional vascular network. In general, the obtained results demonstrate the potential of these systems for bone tissue engineering applications.
\end{abstract}

\section{Introduction}

Tissue engineering offers considerable promise in the repair and replacement of diseased or damaged tissue and is being explored as a potential alternative strategy for tissue repair and organ replacement. ${ }^{1}$ One of most common studied approaches for the development of tissue engineered substitutes aimed at the regeneration of bone defects is based on the interaction of three key elements, namely, a scaffold to support cells and tissue growth, a reliable cell source, and bioactive factors, that can enhance cells/tissue response in different ways. ${ }^{2-4}$

Besides the obvious demands of biocompatibility and controlled degradation profile, the scaffold should feature some important and challenging characteristics, such as to enable the vascularization of the construct upon implantation. ${ }^{5-7}$ This is critical for the cells survival and, therefore, for the formation of the neo-tissues toward the implant. An adequate porosity is required to allow the vascular ingrowth into the scaffolds to improve the nutrients and oxygen supply to the cells in the interior of the construct. ${ }^{8-13}$ The incorporation of bioactive factors capable of promoting angiogenesis into the polymer scaffold (directly or with carriers) leads to an accelerated and successful tissue growth/regeneration. ${ }^{12-15}$ In this context, the design of scaffolds, based on biodegradable polymeric controlled release system constitutes a potential strategy to obtain tissue engineering constructs with increased complexity and functionality. $^{7}$

\footnotetext{
* To whom correspondence should be addressed. E-mail: v.espirito.santo@dep.uminho.pt.

†3B's Research Group.

Institute for Biotechnology and Bioengineering.

${ }^{\S}$ Università di Genova.

${ }^{\perp}$ Istituto Nazionale per la Ricerca sul Cancro.
}

The selection of a suitable material and format for the development of a drug delivery system to be used in tissue engineering applications is a difficult task. ${ }^{16} \mathrm{~A}$ variety of both synthetic and natural polymers have been extensively studied as base materials for obtaining bioactive molecules carriers. ${ }^{17,18}$

To achieve a successful release of growth factors in a tissue engineering approach, one must consider a number of parameters: ${ }^{10,19-21}$ (i) the released growth factors must target the desired cell population; (ii) once they are released, they experience extremely short plasma circulation times and rapid renal clearance; (iii) in general, tissues should be exposed for relatively long periods to these molecules to obtain the proposed effect. ${ }^{15,19,22}$ In summary, a spatiotemporal controlled release system for delivery of growth factors is recommended. ${ }^{4,19}$

Nevertheless, developing an encapsulated protein formulation has been a difficult process because proteins lose their stability quite easily during the processing of the carrier. ${ }^{23,24}$ In this context, hydrogels have emerged as relevant protein carrier systems because they can offer mild processing conditions and hydrophilic environment to the encapsulated protein, therefore, decreasing their potential denaturation and aggregation. ${ }^{25}$

Synthetic polymers, known for their processability have been applied in this field of research, especially saturated poly- $\alpha$ hydroxy esters, including poly(lactic acid) (PLA) and poly(glycolic acid) (PGA). ${ }^{6,26}$ However, many complications have been associated to these polymers from the creation of an acidic environment upon degradation, a feature that is clearly inappropriate for a protein-delivery strategy. ${ }^{27}$ On the other hand, natural polymers have been attractive for this field because they present better biological responses besides a closest-mimicking strategy for a tissue replacement. Some polysaccharides including starch, alginate, hyaluronic acid, and chitosan have been 
used for the development of porous scaffolds and hydrogels for protein and cell encapsulation. ${ }^{6}$

Carrageenan, a naturally occurring polysaccharide shows interesting properties for the development of a controlled release strategy, mainly due to its gelling abilities. Their linear primary structure is based on alternating copolymers of 1,3-linked $\beta$-Dgalactose and 1,4-linked $\alpha$-D-galactose, with varying degrees of sulfatation. The units are joined by alternating $\alpha-1,3$ and $\beta-1,4$ glycosidic linkages forming the disaccharide repeating unit of carrageenans. ${ }^{28-30}$ Due to their half-ester sulfate moieties, carrageenans are strongly anionic polymers. The most common types of carrageenan are traditionally labeled kappa $(\kappa)$, iota $(\gamma)$, and lambda $(\lambda){ }^{31}$ The structures of the three main forms of carrageenan differ only in the number of sulfate groups per disaccharide: $\kappa$ has one, $\gamma$ has two, and $\lambda$ has three. ${ }^{32,33}$ Carrageenan has been increasingly used in the cosmetic and pharmaceutical industries. ${ }^{28}$ The carrageenan biocompatibility has also been discussed in several studies and when used in adequate concentrations, it does not induce a toxic reaction. ${ }^{34}$

Upon cooling under appropriate salt conditions, both $\gamma$ - and $\kappa$-types undergo coil-helix conformational transitions which may lead to gelation, developing ionotropic and thermotropic gels. ${ }^{35}$ Gelation of $\gamma$-carrageenan is enhanced mainly by calcium and $\kappa$-carrageenan by potassium ions. $\gamma$-Carrageenan is either hot or cold soluble and forms soft elastic gels, while the $\kappa$-type dissolves only when heated and forms strong gels that tend to be firm and brittle. ${ }^{32,36,37}$

According to the above-described gelation properties of the main types of carrageenan, it is presumed that the $\kappa$-form could be the most appropriate type to be applied in the development of new carrier formulations, to be integrated in a global tissue engineering strategy to promote bone regeneration.

The entrapment of proteins in an ionotropically cross-linked biodegradable hydrogels may improve the stability of the protein and make it less prone to interference of various formulation excipients. $^{22,38}$

Platelet derived growth factor (PDGF) exists in five isoforms and plays an important role in the stimulation of angiogenesis in vivo. Although it does not directly stimulate endothelial cell proliferation, it acts as a potent mitogen for smooth muscle cells (SMCs) and fibroblasts, stimulating SMCs to produce direct angiogenic factors like vascular endothelial growth factor (VEGF) and basic fibroblast growth factor (bFGF). It is also thought to recruit pericytes and induce the differentiation of endothelial precursor cells. ${ }^{11,24,39-42}$ Besides that, PDGF exerts one of the strongest chemotactic effects on osteoblasts and stem cell precursors. ${ }^{43-45}$ The PDGF-BB isoform is one of the most active in these physiological events; therefore, its choice for encapsulation within the developed hydrogel.

We hypothesized that carrageenans constitute a versatile system in the development of new formulations for the loading and release of different types of protein-based agents as different types of this polysaccharide may be combined. This could be used to adjust the cross-linking density and the charge of the gels to control the release profile of the entrapped bioactive agent.

\section{Materials and Methods}

In this work it is proposed the design of a biodegradable polymeric carrier system based on carrageenan for the sustained delivery of bioactive factors, to promote angiogenesis in a tissue engineering approach. For that, the release profile of PDGF-BB encapsulated in $\kappa$-carrageenan beads was evaluated and some other parameters such as encapsulation efficiency, protein distribution, and cytotoxic evaluation were performed.

2.1. Beads Preparation. Concentrated $\kappa$-carrageenan (22048, SigmaAldrich) solutions in distilled water were prepared by heating the dispersion at $60{ }^{\circ} \mathrm{C}$ with constant stirring to obtain homogeneous solutions $(1.5$ and $2 \%(\mathrm{w} / \mathrm{v}))$. The beads were prepared using an ionotropic gelation method. Briefly, $5 \mathrm{~mL}$ of the previously heated carrageenan solution were extruded dropwise through a syringe needle (0.8 mm in diameter) using a pump (AL-1000, Alladin Programmable Syringe Pump), into $40 \mathrm{~mL}$ of a $\mathrm{KCl}$ (131494.1211, Panreac) solution. Two batches of the resulting beads were allowed to harden for defined periods in the cross-linking solution, while a third batch was removed after a short period. The gel beads were filtered, washed with distilled water twice, and dried in an oven at $37{ }^{\circ} \mathrm{C}$ until constant weight is achieved. Different types of processing parameters were varied, namely, the polysaccharide concentration $(1.5 \%$ or $2 \%(\mathrm{w} / \mathrm{v}))$, the $\mathrm{KCl}$ concentration of the precipitation bath $(1 \%$ or $5 \%(\mathrm{w} / \mathrm{v}))$, and the hardening time in this solution $(\sim 2,15$, and $60 \mathrm{~min})$.

2.2. Characterization of the Developed Beads. To evaluate the potential of the developed $\kappa$-carrageenan beads and establish the formulation/conditions that lead to the systems with the most promising properties for the desired application, the systems were extensively characterized by analyzing their morphology, water uptake, and mechanical stability.

2.2.1. Beads Morphological Characterization. The surface morphology of beads was visualized by stereomicroscope (Zeiss - Stemi 2000-C KL 1500 LCD, 459315) and environmental scanning electron microscopy (ESEM-EDS, FEI Quanta 400, U.K.). For ESEM analysis, the samples were evaluated in their swollen form under $12 \mathrm{mBar}$ of pressure. The internal structure was assessed by cryogenic scanning electron microscopy (JEOL JSM 6301F equipped with a Gatan Alto 2500 EDS).

2.2.2. Mechanical Stability. A determined amount of beads (10 beads/well) were suspended in $1 \mathrm{~mL}$ of PBS solution ( $\mathrm{pH}$ 7.4) with $0.02 \%(\mathrm{w} / \mathrm{v})$ sodium azide in a six-well culture plate. The plate was placed on a thermostatic water bath, at $150 \mathrm{rpm}$ and $37^{\circ} \mathrm{C}$. After 1, 2, $6,24,48$, and $168 \mathrm{~h}$, the beads were collected and observed under a stereomicroscope to count the disrupted beads.

2.2.3. Water Uptake. The swelling ability of the $\kappa$-carrageenan beads was determined by accurately weighing an amount of dry beads and

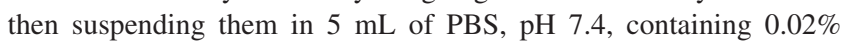
(w/v) sodium azide at $37{ }^{\circ} \mathrm{C}$. After predetermined intervals, the beads were collected by filtration, the excess water was blotted from the surface with filter paper, and the particles were weighed immediately. Eq 1 was used to determine the swelling degree

$$
\% \text { water uptake }=\frac{\left(m_{i}-m_{0}\right)}{m_{0}} \times 100
$$

where $m_{i}$ is the mass of the swollen beads and $m_{0}$ is the initial mass of the original dry beads.

2.3. Loading of the Model Protein (OVA) and the Growth Factor (PDGF-BB). The incorporation of protein molecules into the beads was performed and the efficiency of encapsulation was evaluated. Albumin from chicken egg white (OVA) (A5503-5G, Sigma, molecular weight $42.7 \mathrm{KDa}$, pI 4.54) was selected as the model protein for these studies because its molecular weight is within the same range as the growth factors'.

2.3.1. Encapsulation Efficiency. For the preparation of protein-loaded $\kappa$-carrageenan beads, different formulations with distinct theoretical protein loadings of model protein OVA, and PDGF-BB (100-14B, PeproTech, molecular weight $24.3 \mathrm{KDa}, \mathrm{pI} 10.1)$ were prepared. The protein solution was mixed with the $\kappa$-carrageenan solution in order to obtain a homogeneous distribution and the following procedure was the same as described in section 2.1.

For the model protein, the OVA theoretical loadings were 5 and $10 \%(\mathrm{w} / \mathrm{w})$ with respect to the weight of $\kappa$-carrageenan for each formulation. In the case of the growth factor, the theoretical loading referred to the weight of carrageenan was $0.01 \%$. 
Encapsulation efficiency was determined by an indirect procedure. After the beads preparation process, they were separated from the suspension medium by filtration and the aqueous phase was collected to quantify the protein content in this solution. The nonloaded free protein was determined by the micro-BCA (23235, Pierce) and ELISA (900-K04, PeproTech) kit assays for OVA and PDGF-BB quantification, respectively. Absorbances were measured in a microplate reader, according to each protocol. A calibration curve was made using the supernatant of blank beads. Encapsulation efficiency is calculated by eq 2

$$
\text { \%encapsulation efficiency }=\frac{\left(m_{\mathrm{pt}}-m_{\mathrm{ps}}\right)}{m_{p t}} \times 100
$$

where $m_{\mathrm{pt}}$ corresponds to the initially encapsulated mass of protein, $m_{\mathrm{ps}}$ is the mass of protein in the $\mathrm{KCl}$ solution.

2.3.2. Fourier Transform Infrared Spectroscopy. Fourier transform infrared spectroscopy (IR-Prestige-21, FTIR Shimadzu) was used to record the infrared spectra of $\kappa$-carrageenan, OVA, empty $\kappa$-carrageenan beads, $\kappa$-carrageenan beads encapsulating OVA, and physical mixture of polymer and protein using $\mathrm{KBr}$ pellets. The frequency range covered was $400-4400 \mathrm{~cm}^{-1}$ with a minimum of 36 scans and a resolution of $4.0 \mathrm{~cm}^{-1}$.

2.3.3. Protein Distribution. For visualizing the protein distribution in the beads, OVA was labeled with fluorescein isothiocyanate (FITC; 46951, Sigma). Briefly, the OVA solution was prepared by dissolving the protein in sodium carbonate buffer (S4132-500G, Sigma), $0.1 \mathrm{M}$, $\mathrm{pH} 9$, at a concentration of $10 \mathrm{mg} / \mathrm{mL}$. FITC was dissolved in anhydrous dimethyl sulfoxide (DMSO; 34943, Riedel de Haën) at the same concentration in a darkened laboratory, by simply mixing with vortex. Then the FITC solution was slowly added to the protein solution, at a final ratio of $0.015 \mathrm{mg}$ FITC/mg protein under continuous and moderate agitation at room temperature. The mixture was then allowed to react for $8 \mathrm{~h}$ at $4{ }^{\circ} \mathrm{C}$ in the dark under continuous shaking. The solution was then transferred to a preswollen dialysis membrane tube and after several exchanges of PBS, the dialyzed protein solution was lyophilized. The obtained FITC-labeled OVA was incorporated into the $\kappa$-carrageenan beads by the procedure described in sections 2.1 and 2.3.1. The protein distribution in the beads was examined by fluorescence stereomicroscopy (Olympus SZX16).

2.3.4. In Vitro OVA and PDGF-BB Release Studies. Approximately $100 \mathrm{mg}$ of the dried beads encapsulating OVA and PDGF-BB were suspended in a glass apparatus with $5 \mathrm{~mL}$ of PBS, pH 7.4, containing $0.02 \%(\mathrm{w} / \mathrm{v})$ sodium azide as bacteriostatic agent. The vials containing the bead suspensions were kept at $37{ }^{\circ} \mathrm{C}$ in a shaking water bath at $150 \mathrm{rpm}$. At predetermined intervals, aliquots $(1 \mathrm{~mL})$ of the supernatant were withdrawn and frozen at $-20{ }^{\circ} \mathrm{C}$ for further protein quantification and were replaced with an equal volume of fresh buffer solution to maintain the total volume constant during the release profile study. The supernatant was used to determine the amount of the released protein. The protein quantifications were performed using the micro-BCA assay kit and ELISA for OVA and PDGF-BB determinations, respectively.

The weight of the beads was selected to give a final protein concentration of less than $10 \%$ of the saturation concentration in the medium to ensure sink conditions, which were maintained throughout the whole study.

2.3.5. Sodium Dodecyl Sulfate-Polyacrylamide Gel Electrophoresis (SDS-PAGE). SDS-PAGE of the released model protein was performed using a $10 \%$ acrylamide resolving gel, to evaluate protein molecular weight after the encapsulation and release process (each run contained $15 \mu \mathrm{L}$ of the initial mixture: $15 \mu \mathrm{L}$ of OVA $1 \mathrm{mg} / \mathrm{mL}$ and 8 $\mu \mathrm{L}$ of SDS buffer). The samples were heated at $95{ }^{\circ} \mathrm{C}$ for $7 \mathrm{~min}$. The gel was stained with copper coloration $\left(15 \mathrm{~min}\right.$ in a $0.3 \mathrm{M} \mathrm{CuCl}_{2}$ solution).

2.4. Cytotoxicity Evaluation. To assess the carriers' cytotoxicity, one formulation was chosen: $2 \%(\mathrm{w} / \mathrm{v}) \kappa$-carrageenan precipitated in $5 \% \mathrm{KCl}$ hardened for $60 \mathrm{~min}$. This was the tested condition because it showed adequate morphological features and protein release profiles

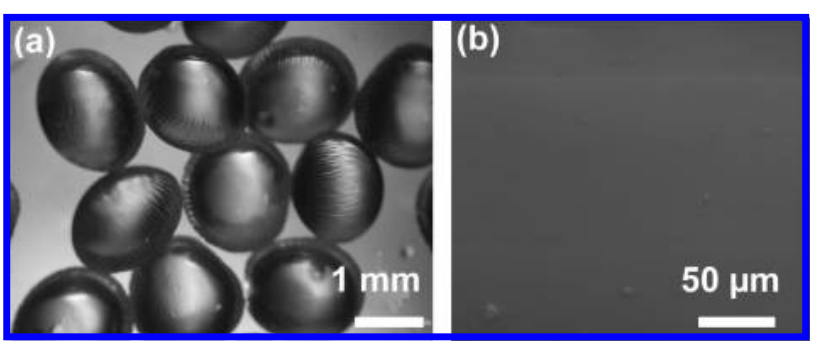

Figure 1. Microscopic images of representative batches of $\kappa$-carrageenan beads obtained by the formulation: $2 \%(\mathrm{w} / \mathrm{v}) \kappa$-carrageenan precipitated in $5 \%(\mathrm{w} / \mathrm{v}) \mathrm{KCl}$ and hardened for $15 \mathrm{~min}$. (a) Stereomicroscopy image of representative beads. The particles present a spherical shape and homogeneous dimensions. (b) Environmental scanning electronic microscopy image of the beads' smooth surface morphology.

matching the goal of the study. For the biological tests, the carrageenan solution was sterilized at high temperatures prior to the beads preparation.

The cytotoxicity tests were performed according to ISO/EN 109935 guidelines. ${ }^{46}$ For the extract preparation, an amount of 100 beads were incubated in $15 \mathrm{~mL}$ (according to the surface area/volume ratio established in the referred guidelines) of culture medium for $24 \mathrm{~h}$ at $37{ }^{\circ} \mathrm{C}$ with constant shaking $(60 \mathrm{rpm})$. As negative control for cytotoxicity behavior, latex extracts were used, prepared in the same conditions as described for the test samples. All the extracts were filtered (using a syringe filter of $0.45 \mu \mathrm{m}$ pore size) to eliminate the possible presence of solid particles of the material.

The cells used in this study were an immortalized cell line of subcutaneous areolar fibroblasts from mouse origin purchased from Collection of Cell Culture (ECACC, U.K.; ref 85011425), designated L929. These cultured cells were tripsinized, resuspended in culture medium at a density of $2 \times 10^{4}$ cells $/ \mathrm{mL}$ and plated $(200 \mu \mathrm{L} / \mathrm{well})$ in 96-well plates at $4 \times 10^{3}$ cells/well. The plates were incubated for $24 \mathrm{~h}$ at $37{ }^{\circ} \mathrm{C}$ in a $5 \% \mathrm{CO}_{2}$ humidified incubator. After that, the medium was replaced by the prepared extracts, using culture medium by itself as positive control, and cultured for 1, 3, 7, and 10 days. At each time point, the culture medium was replaced by MTS solution, $300 \mu \mathrm{L} /$ well (Promega), and incubated for $3 \mathrm{~h}$ at $37{ }^{\circ} \mathrm{C}$ in a $5 \% \mathrm{CO}_{2}$ humidified incubator. MTS solution was transferred to new 96-well plate and absorbance read at $490 \mathrm{~nm}$. All materials were tested in six replicates for each extract for three independent experiments.

2.5. Statistical Analysis. All the experiments were performed with at least three replicates. Results are expressed as mean \pm standard deviation. Differences between the experimental results were analyzed according to a Student $t$-test, with the limit for statistical significance being defined as $p<0.01$.

\section{Results and Discussion}

3.1. $K$-Carrageenan Beads Preparation and Characterization. It was possible to obtain beads using all the described formulations. The obtained beads exhibit a smooth surface and a homogeneous spherical shape with their dimensions ranging between 1 and $2 \mathrm{~mm}$, as it was possible to observe using stereomicroscopy (Figure 1a) and ESEM (Figure 1b). Nevertheless, some of the beads presented lateral rugosity. The spherical shape of the hydrated ones was usually kept after the drying process, especially for the highly cross-linked beads.

CryoSEM allows the investigation of the morphology of hydrated samples preventing the modification of its internal structure. One of the CryoSEM fractures performed on the $2 \%$ $\kappa$-carrageenan beads, precipitated in $5 \% \mathrm{KCl}$ for $15 \mathrm{~min}$, is displayed in Figure 2a, revealing significant differences between the morphology of the beads' surface and their internal structure. In fact, it can be observed an increase in the porosity from the 


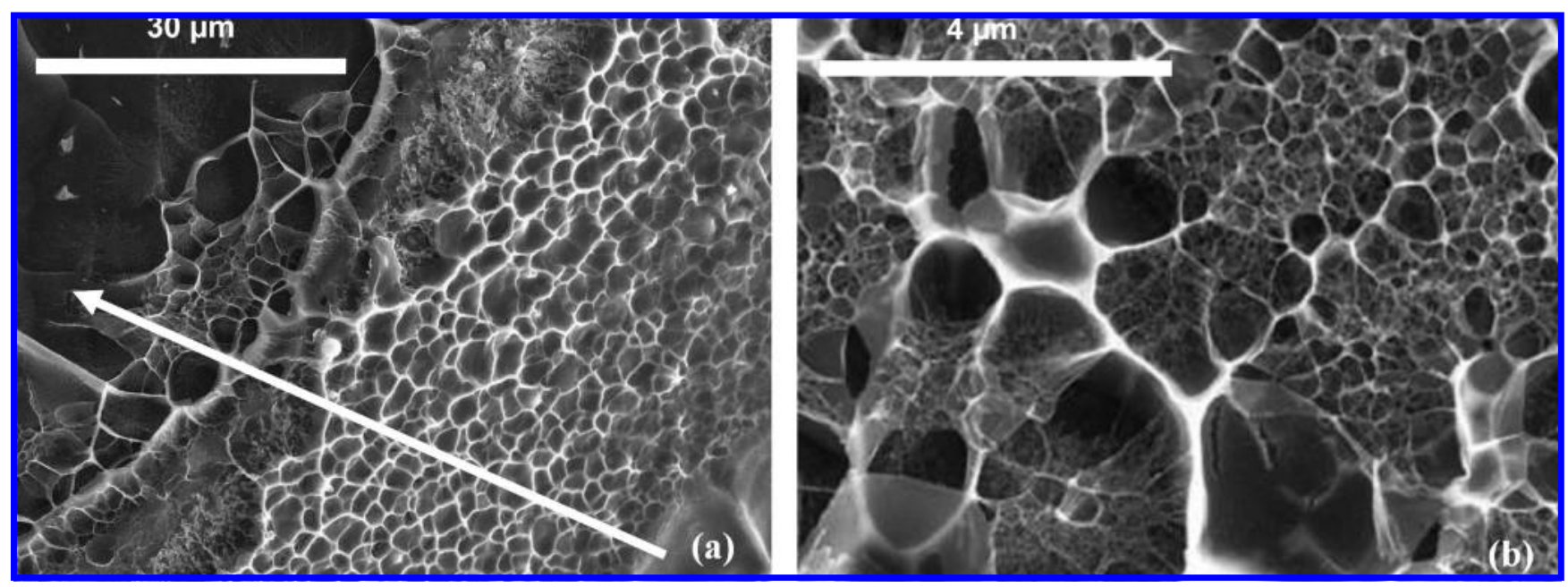

Figure 2. Cryo-SEM images of the surface fracture from beads prepared with $2 \%(\mathrm{w} / \mathrm{v}) \kappa$-carrageenan in $5 \%$ (w/v) $\mathrm{KCl}$ for $15 \mathrm{~min}$ : (a) a crosslinking density gradient is observed in the surface fracture of the bead (the arrow is directed from the surface to the inner core of the bead). The network in the outer layers of the bead indicates a higher degree of cross-linking from the surface to the interior; (b) a magnified image of the inner core of the bead. The hydrogel-like porosity dominates the structure.

right to the left side of the image, that is, from the surface to the interior of the bead. Therefore, the inner areas of the bead show a more disorganized structure and interconnected porosity, with pores with sizes between 1 and $2 \mu \mathrm{m}$. However, a nanostructure phase exhibiting a fibrilar texture may be seen in the interior of such voids (Figure $2 b$ ).

The increase in porosity from the surface to the inner core of the beads results from a higher degree of cross-linking in the outer layers of the beads. When the $\kappa$-carrageenan solution drops from the syringe to the hardening solution, instantaneous interfacial cross-linking takes place followed by a more gradual gelation of the interior.

In addition, the influence of some parameters such as $\kappa$-carrageenan concentration, $\mathrm{KCl}$ solution concentration, and hardening time on the structure of the obtained $\kappa$-carrageenan beads was evaluated. In general, it was found from macroscopic observations that the increase in the $\kappa$-carrageenan concentration, $\mathrm{KCl}$ solution concentration, and hardening time improved the long-term maintenance of the spherical shape of the beads. Although the beads prepared from the $2 \%(\mathrm{w} / \mathrm{v}) \kappa$-carrageenan concentrations seemed to be stronger than the others, when the mechanical stability assessment was performed, no differences were observed. In fact, all the formulations were resistant to the agitation and only after 1 week of constant shaking it was observed that the beads started to shrink. Both 1.5 and $2 \%$ $(\mathrm{w} / \mathrm{v}) \kappa$-carrageenan solutions were able to produce particles when precipitated in 1 and $5 \%(\mathrm{w} / \mathrm{v}) \mathrm{KCl}$ solutions $(\kappa-$ carrageenan concentrations under those values were not able to produce beads that could sustain their shape). Even with only a short cross-linking period, the particles remained morphologically stable, but some improvements were observed with the increase in the hardening time in $\mathrm{KCl}$ solution. From the different processing combinations analyzed, the conditions corresponding to the precipitation of $1.5 \% \kappa$-carrageenan in $1 \%$ $\mathrm{KCl}$ with short hardening time were unable to produce spherical beads. The lower carrageenan concentration and the reduced cross-linking reaction (due to the short exposure of the beads to a less concentrated $\mathrm{KCl}$ solution) are the main reasons for the instability of this last formulation.

For the following characterization procedures, only the beads prepared with a $2 \%(\mathrm{w} / \mathrm{v}) \kappa$-carrageenan concentration were used as they showed a more stable condition for further analysis and application.
Water uptake is a determinant property for drug delivery studies. In the case of hydrogels, swelling is the most important mechanism to promote the release of the incorporated drug because the penetration of water molecules into the structure of the dry hydrogel promotes the relaxation of the chains and the increase in the pores' size. The swelling degree determines the diffusivity of molecules within the hydrogel and it was determined with PBS as the immersion solution, as this buffer will also be used as the release medium in the following experiments. ${ }^{47}$ The developed $\kappa$-carrageenan beads show an affinity for water absorption that is explained by the presence of highly polar sulfate groups in the polymer structure. ${ }^{37}$

From the water uptake measurements displayed in the Figure 3 , it is possible to observe that a quick burst of water penetration occurs within the first $8 \mathrm{~h}$ of testing. After this sharp increase, the swelling increases in a more controlled manner.

One should expect that the cross-linking degree and polymer concentration should affect the swelling based on their effects on the gel charges and network density. ${ }^{48}$ Figure $3 \mathrm{a}$ and $\mathrm{b}$ shows that the conditions prepared with a short cross-linking period ( $2 \mathrm{~min}$ ) present a higher water uptake percentage. The influence of the hardening solution concentration is also visible as the beads prepared with $5 \% \mathrm{KCl}$ display a lower swelling when compared to the hydrogels hardened in $1 \% \mathrm{KCl}$. In both situations, the crosslinking degree affects the swelling rate in an inverse relationship: as the former increases, the latter is reduced. Degradation studies of the carrageenan beads were also performed (data not shown). The beads were kept in PBS at a water bath programmed at $37^{\circ} \mathrm{C}$ and $60 \mathrm{rpm}$ and it was possible to observe that, during the first days of immersion in PBS, the hydrogels' dry mass (after each time point, the beads were dried at $37{ }^{\circ} \mathrm{C}$ until they achieved a constant weight) decreased up to $30 \%$ of the initial dry mass. This reduction can be attributed to the release of residual cross-linking agent. After this decrease, the dry mass was stable up to 8 weeks, thus showing the capability of the systems to be used for longterm delivery purposes.

3.2. Incorporation and Release of the Model Protein in the $\kappa$-Carrageenan Beads. As previously mentioned, the incorporation of proteins into the beads was first carried out using OVA as a protein model. The encapsulation technique that was used in this study is a considerably fast, simple, and inexpensive method for the entrapment of bioactive molecules. The encapsulation efficiency (EE, \%) obtained for the different prepared formulations 


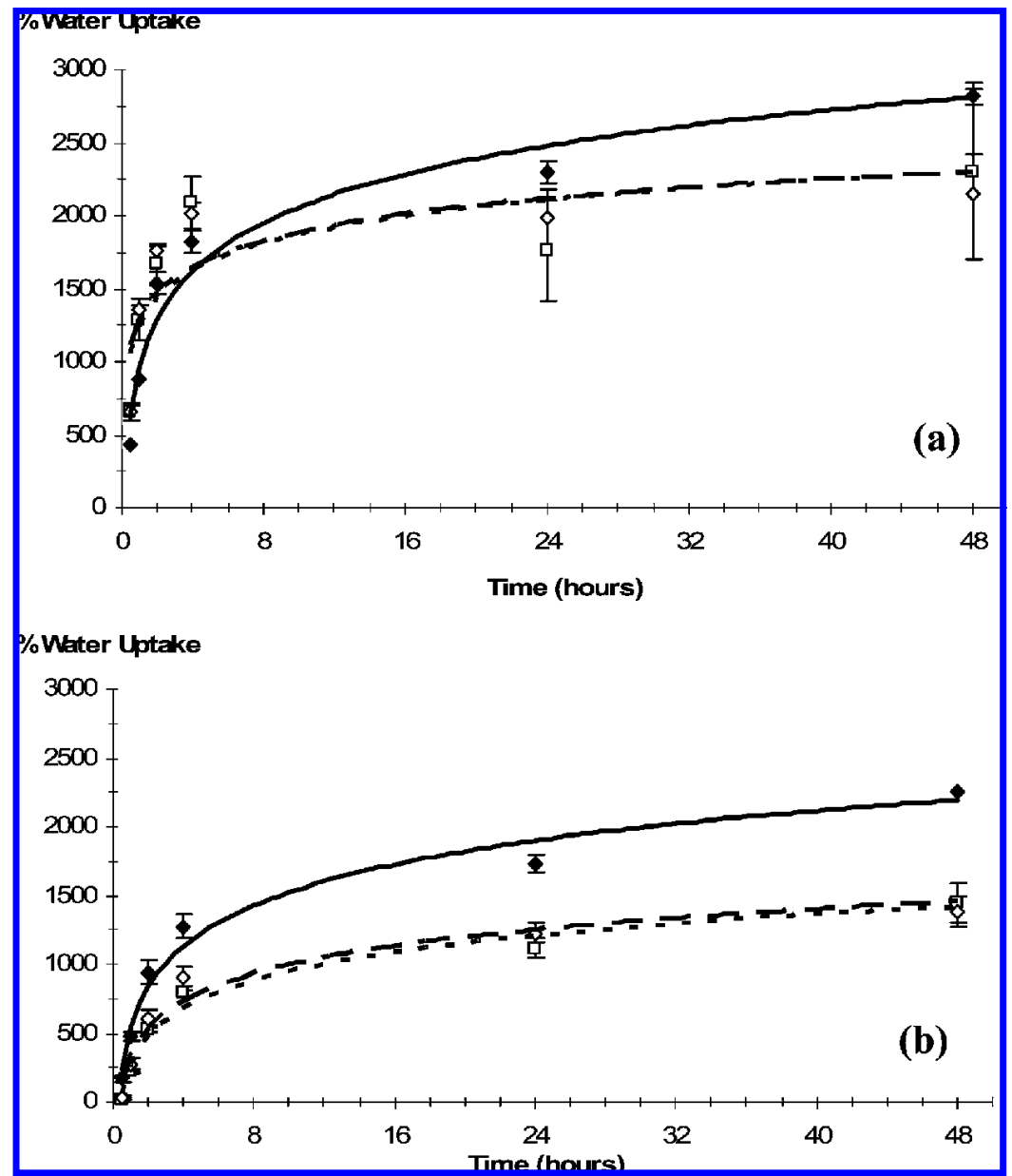

Figure 3. Water uptake studies for different formulations of $2 \% \kappa$-carrageenan beads. (a) Hydrogels prepared with $1 \% \mathrm{KCl}$ cross-linked for different periods: $\diamond$ cross-linking time $(\mathrm{CT})=2 \mathrm{~min}$ with tendency curve $(-)$, $\square \mathrm{CT}=15 \mathrm{~min}$ with tendency curve $(----)$, and $\diamond \mathrm{CT}=60 \mathrm{~min}$ with tendency curve (- -); (b) hydrogels prepared with $5 \% \mathrm{KCl}$ cross-linked for different periods: $\bullet \mathrm{CT}=2$ min with tendency curve (-), $\square \mathrm{CT}=15$ min with tendency curve (----), and $\diamond \mathrm{CT}=60$ min with tendency curve (- -). The effect of $\mathrm{KCl}$ concentration and immersion time in the cationic solution on the swelling ability of the beads was evaluated.

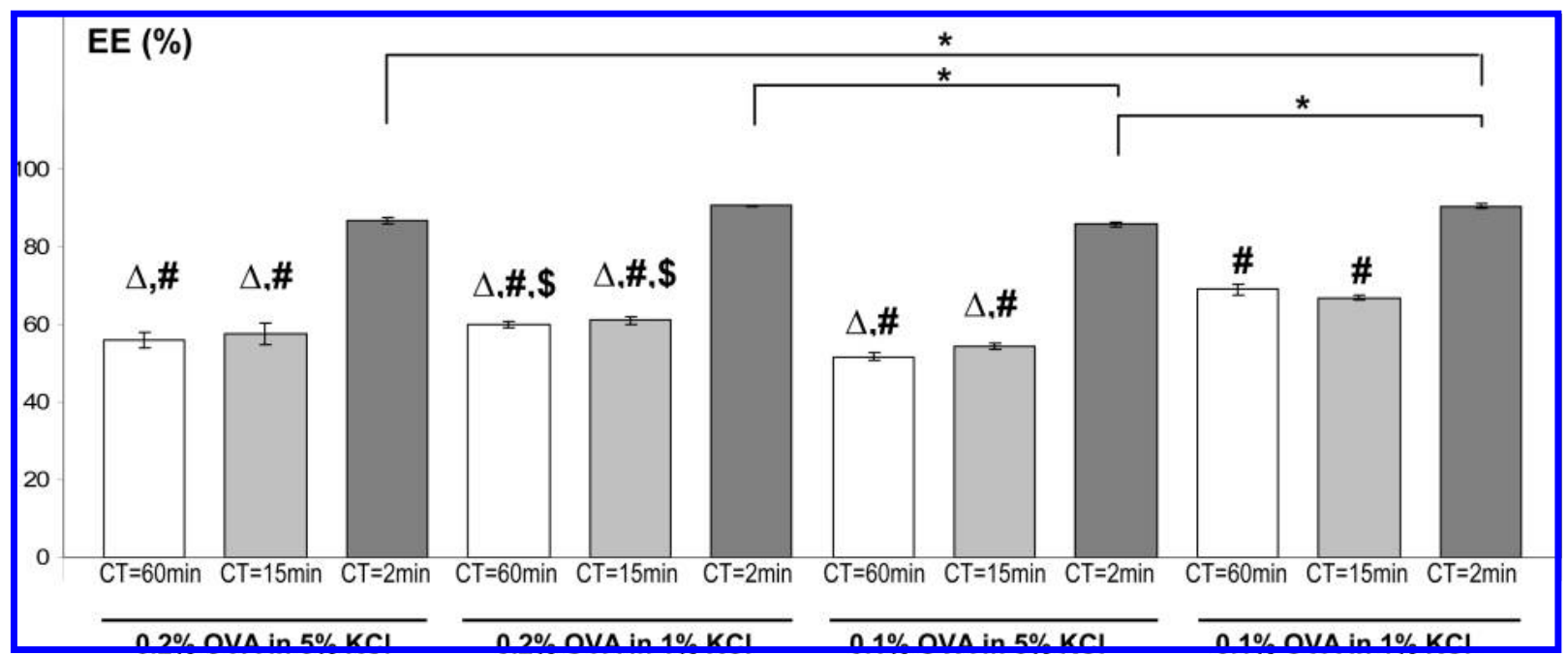

Figure 4. OVA encapsulation efficiency studies for different formulations of $2 \% \kappa$-carrageenan beads. CT, cross-linking time. The formulations showing the symbol \# present statistically significant differences relatively to all systems prepared with a 2 min cross-linking time $(p<0.01) . \Delta$ represents a statistically significant difference $(p<0.01)$ relatively to both formulations: $2 \% \kappa$-carrageenan $+0.1 \%$ OVA in $1 \% \mathrm{KCl}$ for 60 and 15 min. $\$$ represents a statistically significant difference $(p<0.01)$ relative to both designs: $2 \% \kappa$-carrageenan $+0.1 \%$ OVA in $5 \% \mathrm{KCl}$ for 60 and 15 min. The symbol * is used for individual specific significant differences $(p<0.01)$ between beads prepared with a reduced hardening time $(C T=2$ min).

is shown in Figure 4. The results presented in this figure show that all the conditions displayed an EE above $50 \%$.

The nonloaded beads were similar in shape and morphology to the protein-loaded beads. The parameter which seems to be more influent over the encapsulation efficiency is the crosslinking time. When carrageenan beads are developed with a 2 min hardening time, the encapsulation efficiency remains higher than $80 \%$ and is significantly above all the other formulations 
(symbol \#). The protein release during the immersion period justifies the above-mentioned difference as carrageenan sulfate groups are interacting with the $\mathrm{K}^{+}$ions present in the coagulation bath. Independently of the $\mathrm{KCl}$ and protein concentrations, this effect was observed.

In the formulations prepared with $0.1 \%$ OVA, significant differences (symbol $\triangle$ ) are observed between the encapsulation efficiency of particles produced in 1 and $5 \% \mathrm{KCl}$ for the longest cross-linking periods (15 and $60 \mathrm{~min}$ ). Above a critical salt concentration, the anionic carrageenan groups get saturated, which causes a shift from polymer-solvent to polymer-polymer interactions. ${ }^{49,50}$ The expected electrostatic interactions between $\kappa$-carrageenan and the incorporated protein become less intense due to the competition for the sulfate groups. This significant difference is not pronounced in the conditions encapsulating $0.2 \% \mathrm{OVA}$ as this concentration might be a threshold to elicit the differences imposed by the different cross-linker bath conditions. In general, with both OVA loadings ( 0.1 and $0.2 \%$ $(w / v))$, the encapsulation efficiency is lower for the formulations prepared in $5 \% \mathrm{KCl}$ as the competition with the $\mathrm{K}^{+}$ions for the sulfate groups is enhanced. In these cases, a higher fraction of the protein does not get fixed to the gel.

For all the formulations, there is no significance over the encapsulation efficiency between conditions cross-linked for 15 and $60 \mathrm{~min}$.

The FTIR spectra of the different samples were used to identify the typical peaks and to observe if the protein was effectively incorporated into the particulate systems. The FTIR spectra from the different $\kappa$-carrageenan containing samples is presented in Figure 5 and exhibits some identifiable vibrational peaks: the broadband from 3150 to $3600 \mathrm{~cm}^{-1}$ correspond to the hydroxyl group; the band from 2968 to $2850 \mathrm{~cm}^{-1}$ assigned to the $\mathrm{C}-\mathrm{H}$ stretch, the peak at $1640 \mathrm{~cm}^{-1}$ related to polymerbound water, the ester sulfate peak is seen at $1225 \mathrm{~cm}^{-1}$, the peak at $930 \mathrm{~cm}^{-1}$ for 3,6-anhydro-D-galactose and the peak at $845 \mathrm{~cm}^{-1}$ for the D-galactose-4-sulfate. ${ }^{38}$ On the other hand, the OVA spectrum also shows the usual protein peaks, including one wide band between 3190 and $3400 \mathrm{~cm}^{-1}$ due to the $\mathrm{N}-\mathrm{H}$ stretch, one weak peak at $2980 \mathrm{~cm}^{-1}$ due to $\mathrm{C}-\mathrm{H}$ stretch, peaks at 1650 and $1560 \mathrm{~cm}^{-1}$ due to the amide bands I (representing $80 \%$ of the $\mathrm{C}=\mathrm{O}$ stretching vibration of the amide group) and II (mainly $\mathrm{N}-\mathrm{H}$ bending), respectively. The empty beads spectrum was similar to the protein-loaded one, which can be explained by the incorporation of the protein into the carrageenan matrix. The FTIR allows the detection of the established bonds only few micrometers through the beads' surface, therefore, the similarities between both spectra prove the OVA distribution mainly in the hydrogel's core (which converges with the encapsulation efficiency findings). ${ }^{51}$ In the physical mixture of OVA and $\kappa$-carrageenan powder, it is possible to identify peaks correspondent to both samples. These spectrum confirm that the OVA is incorporated in the beads at a molecular level.

Figure 6 resumes the OVA release studies of different formulations. The release profiles were mainly characterized by a burst release within the first few hours of the study (Figure $6 c, d)$. High release rates occur in the systems cross-linked in $\mathrm{KCl}$ for a short period of time $(2 \mathrm{~min})$ due to the presence of the drug at and near the surface. The less cross-linked hydrogels also present higher-dimensioned porous structure, which allows a quicker diffusion of the encapsulated molecules. The interaction between the $\mathrm{K}^{+}$in the coagulation bath and the sulfate groups in the carrageenan structure creates a denser network which is more effective in the entrapment of proteins within the matrix.

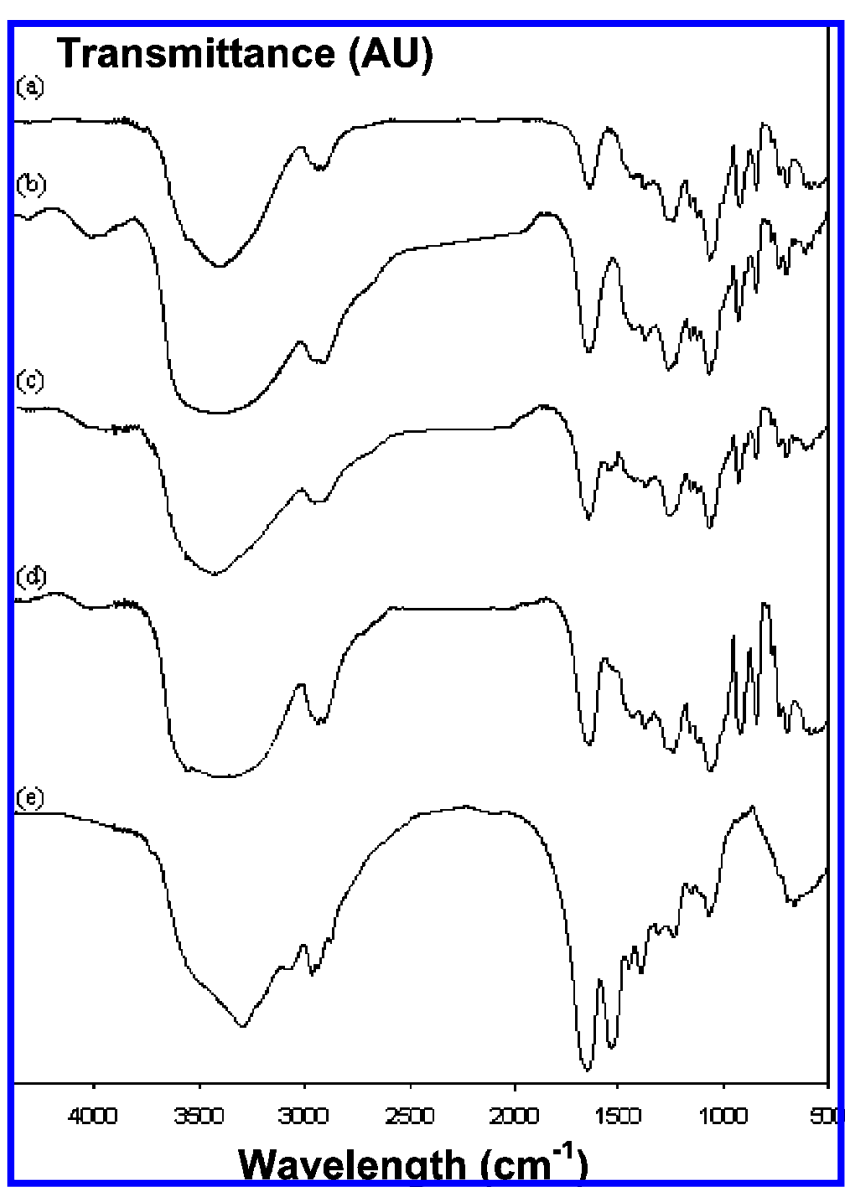

Figure 5. FTIR spectra. Transmittance is shown in arbitrary units (AU) as the analysis is qualitative: (a) $\kappa$-carrageenan powder; (b) $\kappa$-carrageenan blank beads; (c) $\kappa$-carrageenan beads encapsulating OVA; (d) physical mixture of blank beads and OVA; (e) OVA.

However, the beads prepared from the $2 \%(\mathrm{w} / \mathrm{v}) \kappa$-carrageenan solution precipitated in $5 \%(\mathrm{w} / \mathrm{v}) \mathrm{KCl}$ and hardened for 60 min showed a much more controlled release profile. Within the first two days of release, while many other formulations prepared with $5 \% \mathrm{KCl}$ already had released all the protein content, in this case only around 60 and $50 \%$ (for 0.2 and $0.1 \%$ OVA initial loadings, respectively) were quantified in the release medium, maintaining a slow yet controlled release profile during the following days.

The displayed protein release profile from the $\kappa$-carrageenan beads was typical of a hydrogel and the main mechanism involved in this process is the swelling of the beads, which promotes the polymer chain relaxation due to water penetration into the matrix. High release rates take place due to the increased mobility of the protein inside the matrix, allowing its diffusion to the medium. ${ }^{52,53}$ In this particular case, the dissolution of $\kappa$-carrageenan chains that were not cross-linked also seems to contribute to the quick phase of the release profile. This burst seems to be more prominent for the beads prepared with lower cross-linking degrees; in this case, the particles are less dense and the protein is less tight to the matrix. After the burst phase, the release continues with a diffusion mechanism through the matrix with a much slower release rate. In fact, in most of the release profiles a stationary phase is visible, which is probably due to the transition between the release of OVA disposed on the surface of the beads and the release of the OVA that was dispersed in the inner core of the carrier. Therefore, this release profile can be correlated with the beads' structure (shown in Figure 2). The protein content that was dispersed near the surface 


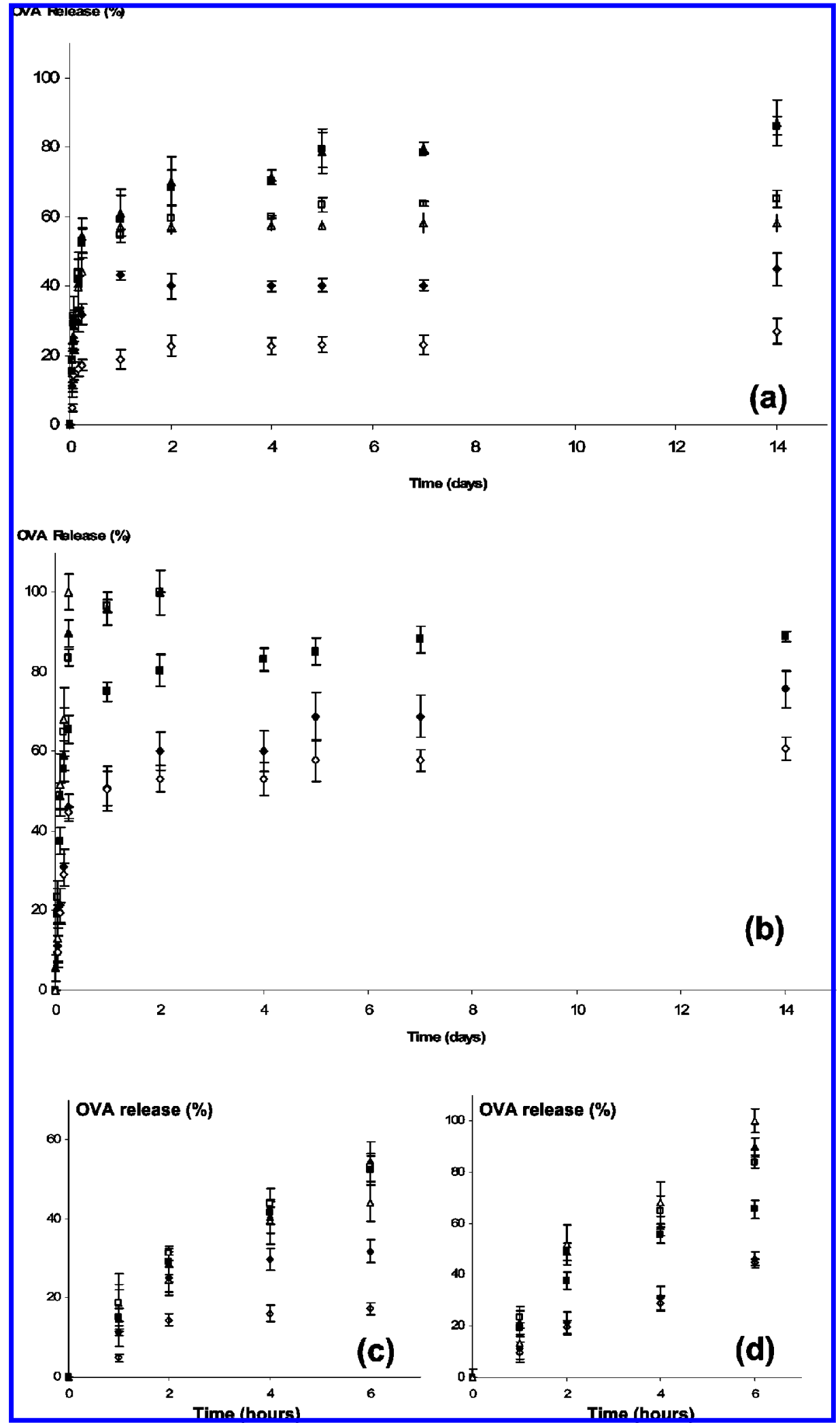

Figure 6. OVA release profiles from carrageenan beads. (a) Formulations represented by the symbols: $\bullet 2 \% \kappa$-carrageenan $+0.2 \%$ OVA in $1 \% \mathrm{KCl} 60 \mathrm{~min} ; 2 \% \kappa$-carrageenan $+0.2 \%$ OVA in $1 \% \mathrm{KCl} 15 \mathrm{~min} ; \boldsymbol{\Delta} 2 \% \kappa$-carrageenan $+0.2 \%$ OVA in $1 \% \mathrm{KCl} 2 \mathrm{~min} ; \diamond 2 \% \kappa$-carrageenan $+0.1 \%$ OVA in $1 \% \mathrm{KCl} 60 \mathrm{~min} ; \square 2 \% \kappa$-carrageenan $+0.1 \%$ OVA in $1 \% \mathrm{KCl} 15 \mathrm{~min} ; \triangle 2 \% \kappa$-carrageenan $+0.1 \%$ OVA in $1 \% \mathrm{KCl} 2 \mathrm{~min}$. (b) The symbols represent the same formulations described in (a) but all disposed prepared in $5 \% \mathrm{KCl}$, all disposed in the same order. (c) OVA release up to $6 \mathrm{~h}$ for the formulations described in (a). (d) OVA release up to $6 \mathrm{~h}$ for the formulations described in (b).

in the denser network was initially released to the medium while the OVA distributed in the core of the bead migrated to the outer layers being released afterward. The controlled profile obtained for the beads cross-linked for $1 \mathrm{~h}$ suggests that an increase of the hardening time might play a key role in the development of a sustained delivery.

The protein loading also seems to affect the release pace as the highest loading formulations $(0.2 \% \mathrm{OVA})$ showed quicker 


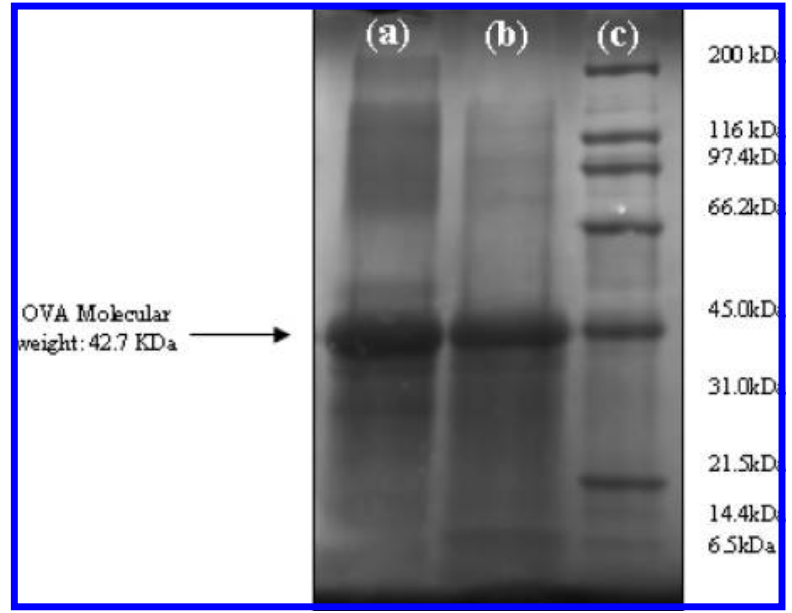

Figure 7. SDS-PAGE of OVA protein: (a) standard OVA, with initial concentration of $1 \mathrm{mg} / \mathrm{mL}$; (b) OVA released from the formulation: $2 \%(\mathrm{w} / \mathrm{v}) \kappa$-carrageenan $+0.2 \%$ OVA precipitated in $5 \% \mathrm{KCl}$ and hardened for $2 \mathrm{~min}$ in that solution. The sample was taken after 2 days of release, when the release had already finished; (c) Marker standard having the indicated molecular masses in KDa.

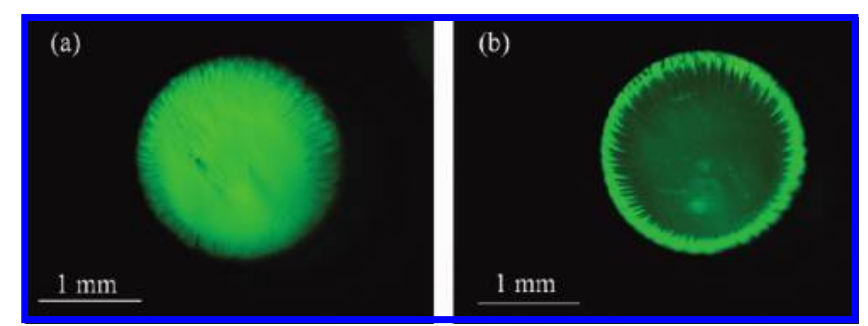

Figure 8. Fluorescence stereomicroscopy images of $2 \% \kappa$-carrageenan $+0.2 \%$ OVA-FITC in $5 \% \mathrm{KCl} 60$ min beads: (a) $\kappa$-carrageenan bead right before the release study initiation; (b) $\kappa$-carrageenan bead after 2 days of release studies.

delivery profiles in the conditions prepared with a high crosslinking time $(60 \mathrm{~min})$. This effect might be explained by the increased protein gradient toward the release media because the beads encapsulate a higher amount of OVA.

SDS-PAGE analysis (Figure 7) was performed to evaluate the molecular weight of the released protein. After 2 days of delivery, the standard OVA run in the electrophoresis gel is quite similar to the encapsulated one.

The protein distribution was evaluated by fluorescence stereomicroscopy, using the beads corresponding to the following formulation: $2 \% \kappa$-carrageenan $+0.2 \%$ OVA-FITC precipitated in $5 \% \mathrm{KCl}$ for $1 \mathrm{~h}$. OVA-FITC was found to be homogeneously distributed on the beads surface (Figure 8a). However, after a 2 day period release, the fluorescence was reduced (Figure $8 \mathrm{~b}$ ), which demonstrates the protein diffusion toward the outer surface of the matrix.

3.3. Incorporation and Release of the Growth Factor in the $\kappa$-Carrageenan Beads. The results obtained from the release studies with the model protein, OVA, suggested that by changing some processing parameters one could obtain different sustained delivery profiles of the molecules; this information can be useful in the development of adequate formulations for the controlled release of growth factors in tissue engineering strategies. However, the release behavior is also influenced by the encapsulated protein, and because of that, the previous conclusions cannot be directly applied to other proteins without testing the system with the desired growth factor. In the proposed system, the penetration of $\mathrm{K}^{+}$ions through the $\kappa$-carrageenan network and the establishment of ionic bonds between the

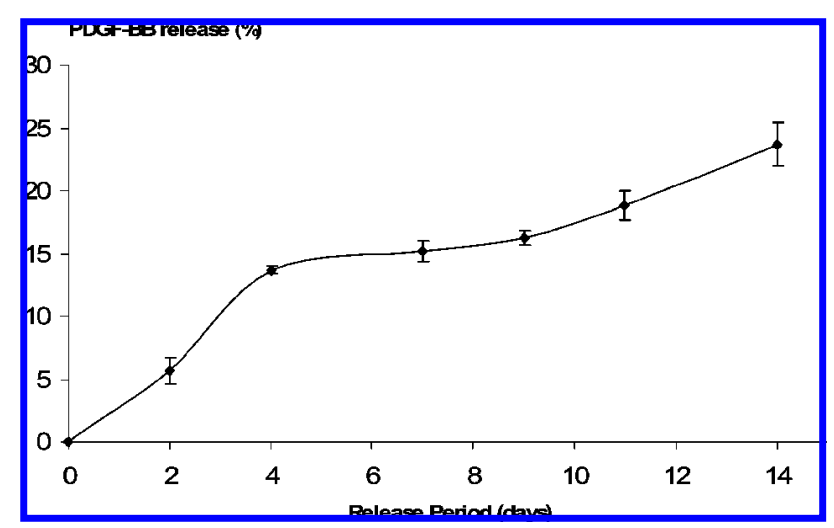

Figure 9. PDGF-BB release from $2 \% \kappa$-carrageenan beads crosslinked in $5 \% \mathrm{KCl}$ for $60 \mathrm{~min}$.

cations and the sulfate groups strengthen the gel, which might be important for the promotion of a slower release. Simultaneously, this effect is responsible for the reduction of encapsulation efficiency, which is a negative feature for yield and economic aspects. Therefore, it is important to find a compromise between an adequate release profile and an advantageous entrapment efficiency, which should be designed according to the target application of the system.

The formulation chosen for PDGF-BB encapsulation and release studies was $2 \% \kappa$-carrageenan in $5 \% \mathrm{KCl} 60$ min because its OVA release profile matches the desired one.

The initial content of PDGF-BB was $0.01 \%$ (w/w) to $\kappa$-carrageenan weight. With the incorporation of PDGF-BB into the $\kappa$-carrageenan matrix, the encapsulation efficiency still remains quite high. The incorporation of PDGF-BB into the carrageenan matrix proved to be quite successful as $74.04 \pm$ $2.77 \%$ of the initial growth factor was encapsulated. This value is higher than the ones with this formulation for the model protein and might be related to the weaker competition between $\mathrm{K}^{+}$ions and the protein. Moreover, the initial protein loading, lower in the PDGF-BB case, might also contribute to a reduction in the establishment of a diffusion gradient toward the release media.

Once again, the results displayed on Figure 9 show that the release profiles correspond typically to a two step process, in which the first correspond to a burst release that takes place within the first 4 days. The PDGF-BB release from the $\kappa$-carrageenan carriers is apparently slower than the OVA release. The growth factor delivery lasted for all the test period, releasing close to $25 \%$ of the initial protein content within the first 2 weeks, thus, showing a controlled delivery process. The release of PDGF-BB followed a discrete initial burst release, thus, avoiding the more pronounced burst diffusion observed in several other published systems. ${ }^{54-59}$

In this work, the protein is not chemically attached to the polymer. The interactions that occur between the molecules are only electrostatic and hydrophobic. The results suggest that the interactions of PDGF-BB with the carrageenan matrix are stronger than those observed for the case of OVA. The higher isolectric point (pI) of PDGF-BB compared with OVA's is one of the reasons for this difference as the higher resultant electrostatic interactions produced between the $\kappa$-carrageenan molecule and the protein can be responsible for a stronger attachment to the gel structure.

An initial faster growth factor release can be advantageous in some applications as it rapidly stimulates the beginning of relevant biological processes, which are maintained during the slower release phase that usually follows this initial burst. In 


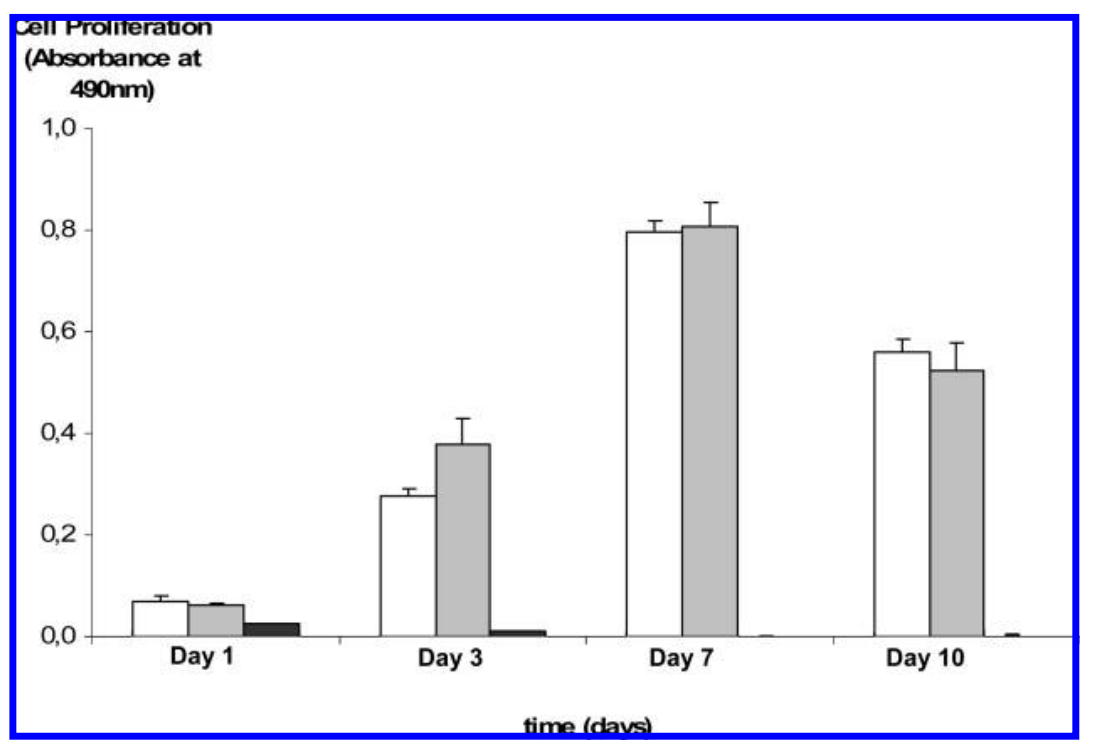

Figure 10. Cell proliferation for the hydrogel formulation: $2 \% \kappa$-carrageenan in $5 \% \mathrm{KCl} 60 \mathrm{~min}$ (white bar), positive control (gray bar), and negative control (latex, black bar). The results are based on the MTS test performed after 1, 3, 7, and 10 days of culture.

several published studies, ${ }^{15,21,40,60-62}$ a continuous $2-4$ week growth factor release is claimed to be the desirable profile, but many other problems are attained in these studies, namely, the bioactivity of the encapsulated bioactive factor during the release study. In this case, the time scale of the release was achieved and after 2 weeks there is still a relevant amount of growth factor entrapped within the carrier matrix, available for release in a latter phase of the study. PDGF-BB is required for the maturation of newly formed blood vessels during the neovascularization process, therefore, this delivery system seems appropriate for this goal.

3.4. Cytotoxicity Assessment. The results obtained from the cytotoxicity (cell viability) assay performed, namely, the MTS test, revealed that the extracts of the tested condition did not affect the viability of the cell line L929 (Figure 10). It is possible to observe that the number of viable cells was similar to the cell number obtained in the positive control. By day 10, cell viability decreases, but this is easily explained by to the cell death induced by space limitations of culture area.

\section{Conclusions}

This study demonstrated that $\kappa$-carrageenan can be an adequate option for the development of carrier systems to encapsulate growth factors or other bioactive agents of relevance in tissue engineering applications, avoiding the use of organic solvents. The high encapsulation efficiency and the controlled delivery profile showed that, with the proper parameters formulation, it is possible to achieve a sustained release of the incorporated bioactive factor. Further cellular studies will be performed to evaluate the biological activity of the released PDGF-BB.

The release profile can be tailored by varying several processing conditions. Moreover, it is expected that the properties of the hydrogels can also be varied by combining different types of carrageenans and by compounding them with other polysaccharides. The release kinetics displayed with these hydrogel beads might be used as a control for other hydrogel shapes. The thermogelling properties of such materials may also be used in new injectable systems that could be used to deliver growth factors and even cells through minimally invasive procedures.
Acknowledgment. This work was supported by the European NoE EXPERTISSUES (NMP3-CT-2004-500283), the European STREP HIPPOCRATES (NMP3-CT-2003-505758), and by the Portuguese Foundation for Science and Technology (FCT) through the project PTDC/FIS/68517/2006 and through the V. Espírito Santo's Ph.D. grant (SFRH/BD/39486/2007).

\section{References and Notes}

(1) Langer, R.; Vacanti, J. P. Science 1993, 260, 920-6.

(2) Yaszemski, M. J.; Payne, R. G.; Hayes, W. C.; Langer, R.; Mikos, A. G. Biomaterials 1996, 17, 175-85.

(3) Rose, F. R.; Oreffo, R. O. Biochem. Biophys. Res. Commun. 2002, $292,1-7$

(4) Tabata, Y. Drug Discoverv Todav 2005, 10, 1639-46.

(5) Finkenzeller, G.; Torio-Padron, N.; Momeni, A.; Mehlhorn, A. T.; Stark, G. B. Tissue Eng. 2007, 13, 1413-20.

(6) Boontheekul, T.; Mooney, D. J. Curr. Opin. Biotechnol. 2003, 14, $559-65$.

(7) Malafaya, P. B.; Silva, G. A.; Baran, E. T.; Reis, R. L. Curr. Opin. Solid State Mater. Sci. 2002, 6, 297-312.

(8) Hutmacher, D. W.; Teoh, S. H.; Zein, I.; Ranawake, M.; Lau, S. Med. Device Technol. 2000, 11, 33-9.

(9) Rezwan, K.; Chen, Q. Z.; Blaker, J. J.; Boccaccini, A. R. Biomaterials 2006, 27, 3413-31.

(10) Lee, K. Y.; Yuk, S. H. Prog. Polvm. Sci. 2007, 32, 669-697.

(11) Patel, Z. S.; Mikos, A. G. J. Biomater. Sci. Polvm. Ed. 2004, 15, 701-26.

(12) Laschke, M. W.; Harder, Y.; Amon, M.; Martin, I.; Farhadi, J.; Ring, A.; Torio-Padron, N.; Schramm, R.; Rucker, M.; Junker, D.; Haufel, J. M.; Carvalho, C.; Heberer, M.; Germann, G.; Vollmar, B.; Menger, M. D. Tissue Eng. 2006, 12, 2093-104.

(13) Mastrogiacomo, M.; Scaglione, S.; Martinetti, R.; Dolcini, L.; Beltrame, F.; Cancedda, R.; Quarto, R. Biomaterials 2006, 27, 3230-7.

(14) Vasita, R.; Katti, D. S. Expert Rev. Med. Devices 2006, 3, $29-47$.

(15) Gu, F.; Amsden, B.; Neufeld, R. J. Controlled Release 2004, 96, $463-$ 72.

(16) Hubbell, J. A. Curr. Opin. Solid State Mater. Sci. 1998, 3, 246-251.

(17) Malafaya, P. B.; Silva, G. A.; Reis, R. L. Adv. Drug Deliverv Rev. 2007, 59, 207-33.

(18) Sokolsky-Papkov, M.; Agashi, K.; Olaye, A.; Shakesheff, K.; Domb, A. J. Adv. Drug Deliverv Rev. 2007, 59, 187-206.

(19) Cao, L.; Mooney, D. J. Adv. Drug Deliverv Rev. 2007, 59, 1340-50.

(20) Lin, C. C.; Metters, A. T. Adv. Drug Deliverv Rev. 2006, 58, 1379408.

(21) Lee, J. Y.; Nam, S. H.; Im, S. Y.; Park, Y. J.; Lee, Y. M.; Seol, Y. J.; Chung, C. P.; Lee, S. J. J. Controlled Release 2002, 78, 187-97.

(22) Mark Saltzman, W.; Baldwin, S. P. Adv. Drug Deliverv Rev. 1998, $33,71-86$.

(23) Putney, S. D. Curr. Opin. Chem. Biol. 1998, 2, 548-52. 
(24) Tessmar, J. K.; Gopferich, A. M. Adv. Drug Delivery Rev. 2007, 59, 274-91.

(25) Coviello, T.; Matricardi, P.; Marianecci, C.; Alhaique, F. J. Controlled Release 2007, 119, 5-24.

(26) Silva, G. A.; Ducheyne, P.; Reis, R. L. J. Tissue Eng. Regener. Med. 2007, 1, 4-24.

(27) Kim, H. K.; Park, T. G. J. Controlled Release 2004, 98, 115-25.

(28) Francis, S.; Kumar, M.; Varshney, L. Radiat. Phvs. Chem. 2004, 69, $481-486$.

(29) Abad, L. V.; Relleve, L. S.; Aranilla, C. T.; Dela Rosa, A. M. $\underline{\text { Radiat. }}$ Phys. Chem. 2003, 68, 901-908.

(30) Chronakis, I. S.; Piculell, L.; Borgström, J. Carbohvdr. Polvm. 1996, 31, 215-225.

(31) Maolin, Z.; Hongfei, H.; Yoshii, F.; Makuuchi, K. Radiat. Phvs. Chem. 2000, 57, 459-464.

(32) Yuguchi, Y.; Urakawa, H.; Kajiwara, K. Food Hydrocolloids 2003, $17,481-485$.

(33) Langendorff, V.; Cuvelier, G.; Michon, C.; Launay, B.; Parker, A.; De kruif, C. G. Food Hydrocolloids 2000, 14, 273-280.

(34) Cohen, S. M.; Ito, N. Crit. Rev. Toxicol. 2002, 32, 413-44.

(35) Chronakis, I. S.; Doublier, J.-L.; Piculell, L. Int. J. Biol. Macromol. 2000, 28, 1-14.

(36) Özbek, H.; Pekcan, Ö. THEOCHEM 2004, 676, 19-27.

(37) Bixler, H. J. Br. Food J. 1994, 96, 12-17.

(38) Sankalia, M. G.; Mashru, R. C.; Sankalia, J. M.; Sutariya, V. B. Int. J. Pharm. 2006, 312, 1-14.

(39) Otrock, Z. K.; Mahfouz, R. A.; Makarem, J. A.; Shamseddine, A. I. Blood Cells, Mol., Dis. 2007, 39, 212-20.

(40) Richardson, T. P.; Peters, M. C.; Ennett, A. B.; Mooney, D. J. Nat. Biotechnol. 2001, 19, 1029-34.

(41) Chen, R. R.; Mooney, D. J. Pharm. Res. 2003, 20, 1103-12.

(42) Lind, M. Acta Orthop. Scand. 1996, 67, 407-17.

(43) Allori, A. C.; Sailon, A. M.; Warren, S. M. Tissue Eng. Part B 2008, 14, 259-73.

(44) Silva, G. A.; Coutinho, O. P.; Ducheyne, P.; Reis, R. L. $\underline{\text { J. Tissue }}$ Eng. Regener. Med. 2007, 1, 97-109.

(45) Silva, G. A.; Coutinho, O. P.; Ducheyne, P.; Shapiro, I. M.; Reis, R. L. Tissue Eng. 2007, 13, 1259-68.
(46) Biological Evaluation of Medical Devices. Part 5: Tests for Cytotoxicity: In Vitro Methods; Standardization, I. O. f., Ed.; Geneva, Switzerland, 1992.

(47) Weng, L.; Gouldstone, A.; Wu, Y.; Chen, W. Biomaterials 2008, 29, 2153-63.

(48) Lu, L.; Mikos, A. G. MRS Bull. 1996, 21, 28-32.

(49) Nickerson, M. T.; Paulson, A. T.; Hallett, F. R. Carbohydr. Polvm. 2004, 58, 25-33.

(50) Sah, H.; Toddywala, R.; Chien, Y. W. J. Controlled Release 1995, 35, 137-144.

(51) van de Weert, M.; van't Hof, R.; van der Weerd, J.; Heeren, R. M.; Posthuma, G.; Hennink, W. E.; Crommelin, D. J. J. Controlled Release 2000, 68, 31-40.

(52) Yeli Zhang, C.-C. C. J. Biomed. Mater. Res. 2002, 59, 318-328.

(53) Sutter, M.; Siepmann, J.; Hennink, W. E.; Jiskoot, W. J. Controlled Release 2007, 119, 301-12.

(54) Hao, X.; Silva, E. A.; Mansson-Broberg, A.; Grinnemo, K. H.; Siddiqui, A. J.; Dellgren, G.; Wardell, E.; Brodin, L. A.; Mooney, D. J.; Sylven, C. Cardiovasc. Res. 2007, 75, 178-85.

(55) Sakiyama-Elbert, S. E.; Das, R.; Gelberman, R. H.; Harwood, F.; Amiel, D.; Thomopoulos, S. J. Hand Surg. 2008, 33, 1548-57.

(56) Gelberman, R. H.; Thomopoulos, S.; Sakiyama-Elbert, S. E.; Das, R.; Silva, M. J. J. Hand Surg. 2007, 32, 373-9.

(57) Liao, I. C.; Wan, A. C.; Yim, E. K.; Leong, K. W. J. Controlled Release 2005, 104, 347-58.

(58) Wei, G.; Jin, Q.; Giannobile, W. V.; Ma, P. X. $\underline{\text { J. Controlled Release }}$ 2006, 112, 103-10.

(59) Park, Y. J.; Ku, Y.; Chung, C. P.; Lee, S. J. J. Controlled Release 1998, 51, 201-11.

(60) Sun, Q.; Chen, R. R.; Shen, Y.; Mooney, D. J.; Rajagopalan, S.; Grossman, P. M. Pharm. Res. 2005, 22, 1110-6.

(61) Murat, Y.; Elçin, V. D. G. G. Artif. Organs 2001, 25, 558-565.

(62) Pieper, J. S.; Hafmans, T.; van Wachem, P. B.; van Luyn, M. J.; Brouwer, L. A.; Veerkamp, J. H.; van Kuppevelt, T. H. J. Biomed. Mater. Res. 2002, 62, 185-94.

BM8014973 